\title{
Control of a Heaving Buoy Wave Energy Converter for Potable Water Production
}

\author{
Gary Nolan and John Ringwood \\ Department of Electrical Engineering \\ National University of Ireland, Maynooth \\ Maynooth, Co. Kildare, IRELAND
}

E-mail: gary.nolan@eeng.nuim.ie john.ringwood@eeng.nuim.ie

\begin{abstract}
This paper details a control strategy for optimal potable water production from a single heaving-buoy wave energy converter with a hydraulic power take-off system.

Keywords - Wave Energy, Power Take-Off, Reverse Osmosis, State Feedback Control.
\end{abstract}

\section{INTRODUCTION}

This paper considers a wave energy converter (WEC) of the heaving buoy type (also known as a point absorber type WEC). The device itself is an offshore floating device which captures energy from its wave induced motion via hydraulic rams and a high pressure power take-off (PTO) system. The captured energy is in the form of seawater under pressure which can be used to produce electricity, via a turbine and electrical generator, or to produce potable drinking water, via a reverse osmosis (RO) desalination system. This paper details the development of a control strategy for a heaving buoy WEC producing potable water only. The RO desalination system purifies pressurised seawater through the use of a semi-permeable membrane, which allows water particles to pass freely through it while rejecting any impurities. The use of wave power for desalination is considered in $[1,2]$; however, this is the first documented control system for such a function, to the best of the authors' knowledge. Fig. 1 is a conceptual diagram of a heaving buoy WEC and its PTO system.

The paper begins with a problem specification, followed by a mathematical description of the full WEC system. The development of the control strategy is discussed before the final control strategy is detailed. Finally, a number of results are illustrated and conclusions are made.

\section{Problem Specification}

The objective of the WEC is to produce as much potable water as possible without compromising the structural integrity of the device and the integrity of its individual components. At the heart

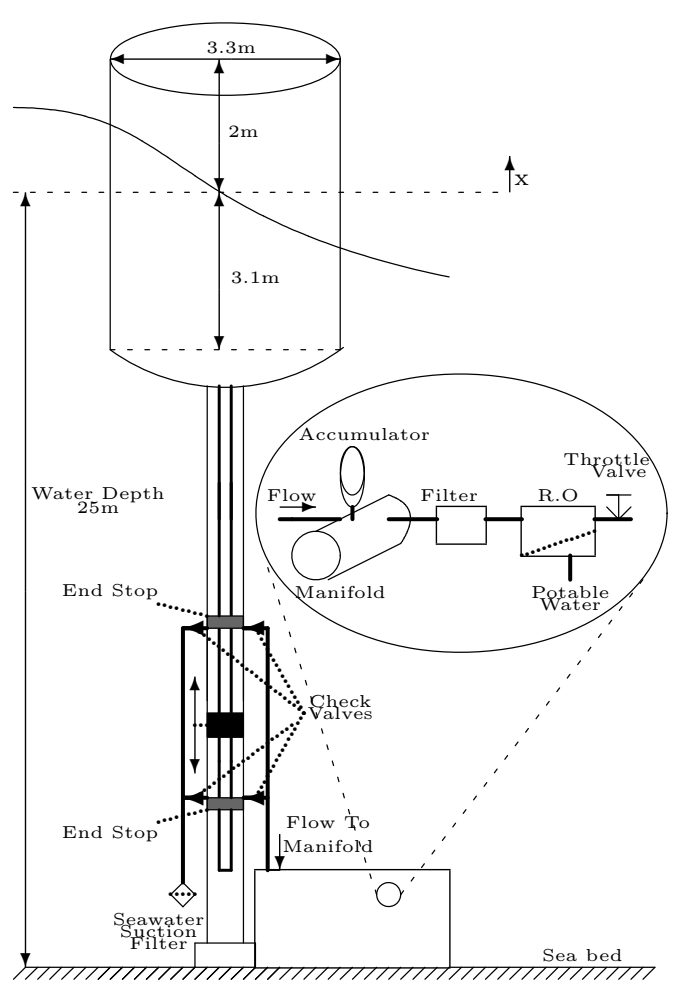

Fig. 1: Conceptual Diagram of a Complete WEC

of maximising the flow of potable water, $Q_{w}$, there is a strict regulation problem. $P_{\text {ro }}(\mathrm{RO}$ process variables illustrated in Fig.2) must be maintained at a specific operating point $\left(6 \times 10^{6} \mathrm{~Pa}\right)$. Any deviation from this pressure will seriously effect the quality of the potable water produced. Even a $3 \%$ difference (positive or negative) will cause the potable water quality to drop rendering it commercially useless [3]. While maintaining this pressure, the ratio of $Q_{t v} / Q_{w}$ must meet or exceed 1.857 


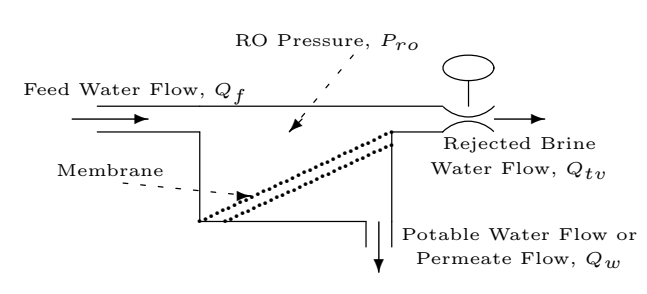

Fig. 2: RO Diagram

in order to avoid salt concentration build up. Finally, there is the facility to switch in and out the number of RO units in operation so as to match the amount of energy the WEC has captured. In summary, maximum potable water production is achieved by having the maximum number of RO units in operation subject to maintaining $P_{\text {ro }}$ at $6 \times 10^{6} \mathrm{~Pa}$ and ensuring $Q_{t v} / Q_{w}$ exceeds 1.857 .

\section{Wave Energy Converter Model}

The WEC model consists of a set of coupled equations: (i) an integral-differential equation containing the hydrodynamics of the buoy, and (ii) an ordinary differential equation (ODE) representing the PTO system. The model incorporates irregular waves, or 'real sea' waves, expressed by the Pierson-Moskowitz (PM) spectrum [4]. The sea state is characterised by $H_{\frac{1}{3}}$ (the significant wave height defined as the mean of the one third highest waves), $T_{1}$ (the mean wave period), and the PM spectrum:

$S(\omega)=\frac{0.11 H_{\frac{1}{3}}^{2} T_{1}}{2 \pi}\left(\frac{\omega T_{1}}{2 \pi}\right)^{-5} e^{\left[-0.44\left(\frac{\omega T_{1}}{2 \pi}\right)^{-4}\right]}$

The predominant wave condition used in this study is characterised by $H_{\frac{1}{3}}=2 \mathrm{~m}$ and $T_{1}=6.5 \mathrm{~s}(\approx$ the nearshore predominant wave condition for the west coast of Ireland).

\section{a) Heaving Buoy Model}

A mathematical model for a heaving buoy [5], for the dimensions given in Fig.1, is given as:

$$
\begin{aligned}
& \ddot{x}(t)=\frac{1}{m_{b}+m_{r}(\infty)}\left\{\int_{-\infty}^{\infty} \eta(\tau) f(t-\tau) d \tau-B(t) \dot{x}(t)\right. \\
& \left.-\int_{-\infty}^{t} r(t-\tau) \dot{x}(\tau) d \tau-R_{f} \dot{x}(t)-S x(t)+F_{m}\right\}
\end{aligned}
$$

where $x(t)$ is the vertical displacement of the buoy and $\eta(t)$ is the wave elevation. A list detailing the parameters in eq. (2) can be found at the end of this section. The kernels or impulse response functions $f(t)$ and $r(t)$ are found by calculating the inverse Fourier transform of the frequency dependant hydrodynamic parameters, $F(\omega)$ and $R(\omega)$ [6]. These parameters are derived numerically using the software package WAMIT (Wave Analysis MIT) [7] and are illustrated in Fig.3. WAMIT also calculates the parameters $m_{r}(\infty)$ and $S$.
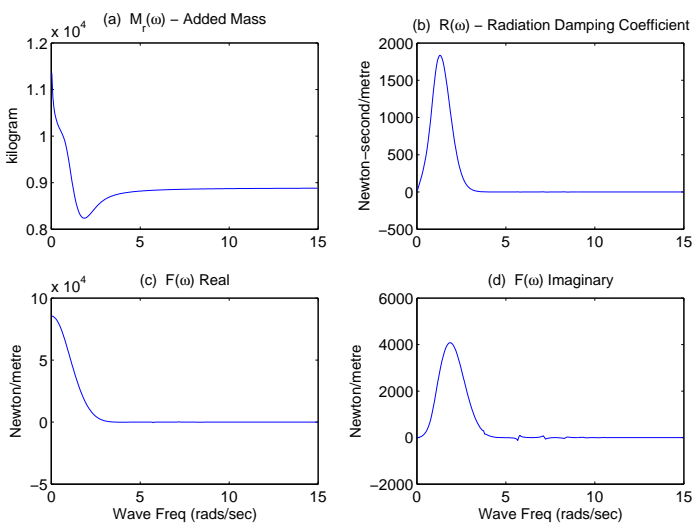

Fig. 3: Hydrodynamic Parameters (from WAMIT)

\section{b) PTO Model}

Using the analogies between hydraulic and electrical systems (pressure $\equiv$ voltage and flow $\equiv$ current), a circuit equivalent of the hydraulic PTO system illustrated in Fig.1 is found (Fig.4). The

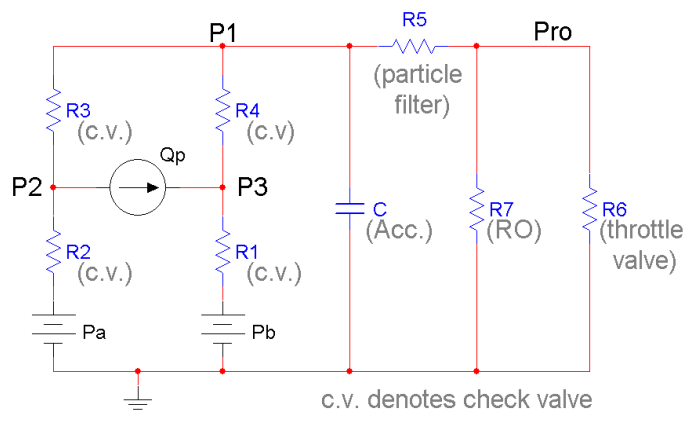

Fig. 4: Circuit Equivalent of the PTO System

current source represents the flow source of the hydraulic pump, the resistive elements represent the valves, filter and RO membrane, and the capacitive element represents the accumulator. The voltage sources, $P_{a}$ and $P_{b}$, represent the pressure at the inlet pipes which force seawater into the hydraulic pump.

Using Kirchoff's current law (KCL) at the nodes $P_{1}, P_{2}, P_{3}$ and $P_{r o}$, the following ODE is determined:

$\dot{P}_{1}=P_{1}\left[-C^{-1}\left(\frac{1}{R_{5}}+\frac{1}{R_{4}}+\frac{1}{R_{3}}-\frac{R_{6} R_{7}}{R_{5} R_{6} R_{7}+R_{5} R_{5} R_{7}+R_{5} R_{5} R_{6}}\right.\right.$

$\left.\left.-\frac{R_{2}}{R_{2} R_{3}+R_{3} R_{3}}-\frac{R_{1}}{R_{4} R_{4}+R_{1} R_{4}}\right)\right]+Q_{p}\left[C^{-1}\left(\frac{-R_{2} R_{3}}{R_{2} R_{3}+R_{3} R_{3}}\right.\right.$

$\left.\left.+\frac{R_{1} R_{4}}{R_{4} R_{4}+R_{1} R_{4}}\right)\right]+P_{a}\left[\frac{R_{3} C^{-1}}{R_{2} R_{3}+R_{3} R_{3}}\right]+P_{b}\left[\frac{R_{4} C^{-1}}{R_{4} R_{4}+R_{1} R_{4}}\right]$

where

$Q_{p}=\dot{x} A_{p}$

$C=v_{0} /\left(p_{0} \gamma\right)$ 
$R_{6}=\frac{d P_{r o}}{d Q_{t v}}=\frac{\sqrt{P_{r o}}}{0.5 C_{v}}$

$R_{7}=\frac{P_{r o}}{Q_{w}}=\frac{P_{r o}}{\left(P_{r o}-P_{o s m}\right) \rho_{r o}}$

The resistance of the various check valves is also calculated using eq. (6), but in the case of the check valves the value of $C_{v}$, the valve flow coefficient, switches between a high value (open) and a low valve (closed). For the throttle valve, $C_{v}$ is a function of valve position and is given by the valves inherent opening characteristic, Fig.7(b).

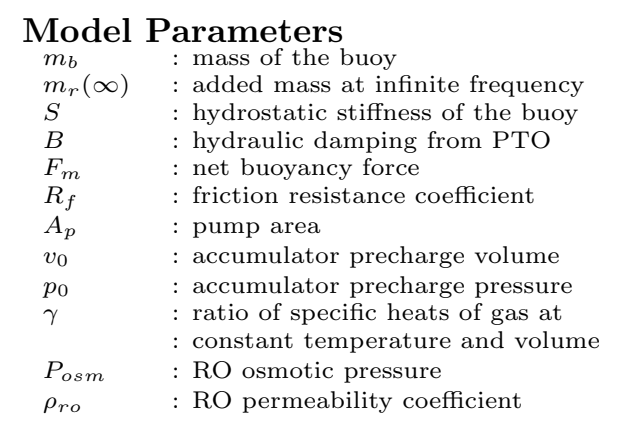

$=9700 \mathrm{~kg}$ $=8700 \mathrm{~kg}$ $=86.4 \mathrm{kN} / \mathrm{m}$

$=173 \mathrm{kN}$ $=200 \mathrm{~kg}$ $=0.009852 \mathrm{~m}^{2}$ $=0.6913 \mathrm{~m}^{3}$

$=2 \times 10^{6} \mathrm{~Pa}$

$=1.4$

$=27 \times 10^{5} \mathrm{~Pa}$

$=1.06 \times 10^{-1}$

\begin{tabular}{|c|c|}
\hline Manipulated Variables & Notation \\
\hline Pos. of throttle valve (\% of stroke) & $V_{\text {pos }}$ \\
Ballast mass & $m_{\text {bal }}$ \\
Number of RO units employed & $N_{R O}$ \\
\hline Controlled Variables & \\
\hline Flow of potable water & $Q_{w}$ \\
Throttle valve flow & $Q_{t v}$ \\
Displacement and velocity of buoy & $x, \dot{x}$ \\
\hline
\end{tabular}

Table 1: Manipulated and Controlled Variables

The throttle valve position, $V_{p o s}$, is the primary MV for control of $P_{\text {ro }}$. While the large accumulator upstream of the RO units smoothes most of the variations in flow and pressure from the hydraulic pump, it is the residual variations in pressure that must be dealt with by the valve and the controller.

Since via the MVs some of the WEC system parameters can be varied, it would be desirable if for the pressure controller a model based control scheme could preserve the algebraic relations between the system parameters and the controller parameters, so that suitable 'adaptation' can be achieved.

\section{Pressure Controller}

The mathematical model of the WEC (eqs. (2) and $(3))$ is nonlinear and contains a number of undesirable convolution products. An attempt at simplification and linearisation of these equations will be made.

\section{a) Model Simplification and Linearisation}

Essentially, eq. (2) is in the form of a simple mass, spring, damper model. Grouping the various expressions for the coefficients of displacement, velocity and acceleration gives:

$M \ddot{x}(t)+\left(B+R_{f}+R_{c}\right) \dot{x}(t)+S x(t)=F(t)$

where $M=m_{b}+m_{r}(\infty)+m_{b a l}$ and $R_{c}$ is the static approximation of $r(t)(=722.1)$ which is found by calculating the area under the kernel. $F(t)$ $\left(=\int_{-\infty}^{\infty} \eta(\tau) f(t-\tau) d \tau\right)$, the wave excitation force, is not approximated as it is only considered as a disturbance for the pressure controller. Finally, $F_{m}$ in eq. (2) is neglected since it only affects $x$ (not controlled) rather than $P_{\text {ro }}$.

The PTO circuit equivalent (Fig.4) can be simplified to the circuit in Fig.6. assuming a current

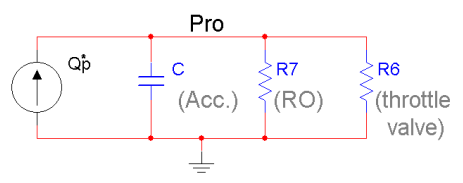

Fig. 6: Simplified Circuit Equivalent of the PTO System source (originally from eq. (4)) given as:

$Q_{p}^{*}=|\dot{x}| A_{p}$ 
giving the rectified flow that comes from the hydraulic pump and its set of check valves.

Also, the nonlinear valve characteristic, Fig.7(b), can be linearised by using the linearising characteristic of Fig.7(a) as a pre-compensator. The resultant linearisation is confirmed by Fig.7(c) with:

$C_{v}=C_{r} V_{\text {pos }}^{*} / 100$

where $C_{r}$ is the rated flow coefficient of the valve.
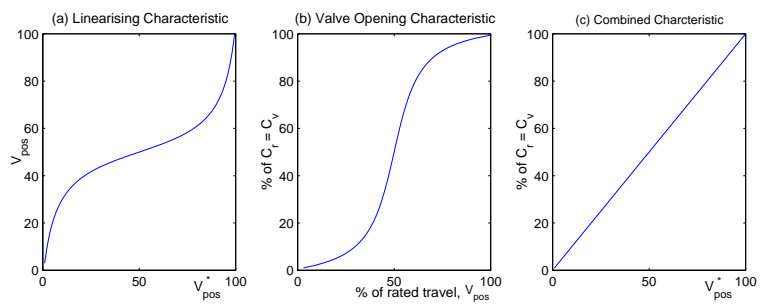

Fig. 7: Valve Characteristic Linearisation

Again using KCL, the simplified PTO ODE, utilising the linearised valve characteristic, is given by:

$\dot{P_{\text {ro }}}=-P_{\text {ro }}\left[\frac{1}{C}\left(\frac{1}{R_{6}}+\frac{1}{R_{7}}\right)\right]+Q_{p}^{*}\left(\frac{1}{C}\right)$

Under conditions of good pressure regulation, $R_{7}$ (eq. (7)) can be considered constant, with $P_{\text {ro }}=\overline{P_{\text {ro }}}=6 \times 10^{6}$. Using the same rationale, the term $\left(P_{\text {ro }} / C R_{6}\right)$ in eq. (13) can be replaced by $\left(C_{r} V_{\text {pos }}^{*} \sqrt{\overline{P_{\text {ro }}}} / C 200\right)$ assuming the linearised valve characteristic. The equation describing the pressure dynamics now becomes:

$\dot{P_{r o}}=\left[\frac{-C_{r} \sqrt{\overline{P_{r o}}}}{200 C}\right] V_{\text {pos }}^{*}-\left[\frac{\left(\overline{P_{r o}}-P_{o s m}\right) \rho_{r o}}{C \overline{P_{r o}}}\right] P_{r o}+\frac{Q_{p}^{*}}{C}$

With $\left[\begin{array}{lll}x_{1} & x_{2} & x_{3}\end{array}\right]^{T}=\left[\begin{array}{lll}x & \dot{x} & P_{\text {ro }}\end{array}\right]^{T}$, the complete system equations can be formulated into a state-space form:

$\dot{x}(t)=\mathcal{A} x(t)+\mathcal{B} u(t)+d(t)$

using eqs. (8), (10) and (14), as:

$$
\begin{aligned}
& {\left[\begin{array}{c}
\dot{x_{1}} \\
\dot{x_{2}} \\
\dot{x_{3}}
\end{array}\right]=\left[\begin{array}{ccc}
0 & 1 & 0 \\
-\frac{S}{M} & -\frac{R_{f}+R_{c}}{M} & -\frac{A_{p}}{M} \\
0 & \frac{A_{p}}{C} & -\left(\frac{\left(\overline{P_{r o}}-P_{o s m}\right) \rho_{r o}}{C \overline{P_{r o}}}\right)
\end{array}\right]} \\
& {\left[\begin{array}{l}
x_{1} \\
x_{2} \\
x_{3}
\end{array}\right]+\left[\begin{array}{c}
0 \\
0 \\
\frac{-C_{r} \sqrt{\overline{P_{r o}}}}{200 C}
\end{array}\right]\left[V_{\text {pos }}^{*}\right]+\left[\begin{array}{c}
0 \\
F(t) \\
0
\end{array}\right]}
\end{aligned}
$$

where

$A_{p}= \begin{cases}0.009852 & , \dot{x}>0 \\ -0.009852 & , \dot{x}<0\end{cases}$

The switching parameter $A_{p}$, carrying the sign of $\dot{x}$, is introduced to eliminate the absolute value function originating in eq. (11). Although, the model now falls into the realms of switching systems and care must be taken to ensure stability is maintained across switching boundaries, the system model is now in a manageable linear state space form which, around the operating point $\overline{P_{\text {ro }}}$, provides an accurate representation of the original model. For example, Fig. 8 contains a plot of $P_{r o}$ from the original model and the linear simplified model with a simple proportional controller regulating $P_{\text {ro }}$ at $\overline{P_{r o}}$.

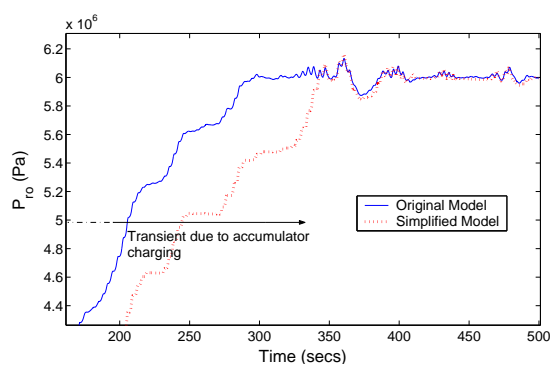

Fig. 8: Sample Plot of $P_{r o}$

\section{b) Stability and Controllability}

Since a common quadratic Lyapunov function (CQLF) exists for the system described by eq. (16) with the switching parameter $A_{p}[9]$, open loop stability is guaranteed [10]. Also, since,

- The transitions between the switched systems are smooth (occurring at $\dot{x}=0$ ),

- The magnitude of the entries in the $\mathcal{A}$ matrix containing the switching parameter are relatively small compared to the other entries, and

- A consistent closed-loop system is achieved,

a strong case for the stability of the closed-loop (system plus feedback controller) switched system is made.

Since the system controllability matrix is of full rank, the system in eq. (16) is fully state controllable.

\section{c) State Feedback Control}

Since a full state feedback controller (SFBC) will attempt to regulate all three states (while $x_{1}$ and $x_{2}$ should remain free), a suitable partition of the state vector is appropriate. Regarding pressure control, the state equation of interest is:

$\dot{x_{3}}=\mathcal{A}_{33} x_{3}+\mathcal{B}_{3} u+\mathcal{A}_{32} x_{2}$

the SFBC is designed such that:

$\dot{x_{3}}=\mathcal{A}_{c} x_{3}+\mathcal{A}_{32} x_{2}$

with $u_{c}=-K x_{3}$ (giving a desired closed loop pole at $\mu_{1}$ ) and $\mathcal{A}_{32} x_{2}$ being used as a disturbance feedforward (DFF) term, with:

$K=\left(\mathcal{A}_{33}-\mu_{1}\right) / \mathcal{B}_{3}$ 
In order to achieve non-zero state (pressure) regulation, the control structure of Fig.9 is introduced and, since $x_{3}$ is the system output, $N_{x}$ and $N_{j}$ can be calculated as:

$\left[\begin{array}{l}N_{x} \\ N_{j}\end{array}\right]=\left[\begin{array}{cc}\mathcal{A}_{33} & \mathcal{B}_{3} \\ 1 & 0\end{array}\right]^{-1}\left[\begin{array}{l}0 \\ 1\end{array}\right]$

Solving for $N_{x}$ and $N_{j}$ gives:

$N_{x}=1, \quad N_{j}=\mathcal{A}_{33} /\left(-\mathcal{B}_{3}\right)$

The DFF from $x_{2}$ should enable a large part of the 'incident wave' effect on $x_{3}$ to be neutralised, with $K_{d}$ (dependent on the switching parameter $A_{p}$ ) in Fig.9 given as:

$K_{d}=\mathcal{A}_{32} / \mathcal{B}_{3}$

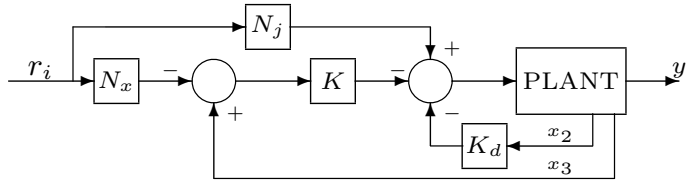

Fig. 9: State Feedback with Disturbance Feedforward Control System

Examination of the (spectrum of the) residual error resulting from the feedforward-only controller allows $\mu_{1}$ to be chosen to maximise the feedback effectiveness. The peak of the residual for the predominant wave condition is approximately 0.12 rads/sec, resulting in the placement of $\mu_{1}$ on the real axis at -0.12 .

Due to the non-zero mean (rectified) wave disturbance and, to a lesser extent, modelling errors, integral action (IA) is introduced to ensure zero steady state error (to constant disturbances/modelling errors). This is achieved through the addition of an extra (integral) state, $x_{I}$, which obeys the state equation:

$\dot{x_{I}}=x_{3}-r_{i}$

The augmented state equations, omitting the disturbance term $\mathcal{A}_{32} x_{2}$, are now:

$\left[\begin{array}{c}\dot{x_{I}} \\ \dot{x_{3}}\end{array}\right]=\left[\begin{array}{cc}0 & 1 \\ 0 & \mathcal{A}_{33}\end{array}\right]\left[\begin{array}{c}x_{I} \\ x_{3}\end{array}\right]+\left[\begin{array}{c}0 \\ \mathcal{B}_{3}\end{array}\right] u-\left[\begin{array}{l}1 \\ 0\end{array}\right] r_{i}$

The new controller gains $\left[\begin{array}{ll}K_{I} & K_{3}\end{array}\right]$ solved from:

$\operatorname{det}\left(s \mathcal{I}-\left[\begin{array}{cc}0 & 1 \\ 0 & \mathcal{A}_{33}\end{array}\right]+\left[\begin{array}{c}0 \\ \mathcal{B}_{3}\end{array}\right]\left[\begin{array}{c}K_{I} \\ K_{3}\end{array}\right]^{T}\right)=\left(s-\mu_{1}\right)\left(s-\mu_{2}\right)$

with both poles placed at $s=-0.12$, are:

$K_{I}=\frac{\mu_{1} \mu_{2}}{\mathcal{B}_{3}} \quad K_{3}=\frac{-\left(\mu_{1}+\mu_{2}\right)+\mathcal{A}_{33}}{\mathcal{B}_{3}}$

The final controller schematic is given in Fig.10.

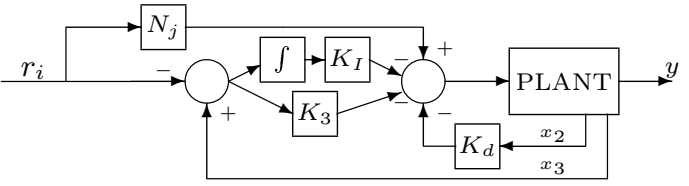

Fig. 10: State Feedback with Integral Action and Disturbance Feedforward Control System

\section{Mass And RO Unit CONTROL}

Items 1 . and 2. of the control hierarchy articulated in section IV are now considered.

With regard to $m_{b a l}$, which allows control of the buoy dynamics through the inertia term $M$ of eq. (10), ballast, in the form of sea water, can be pumped into the buoy in order to tune or de-tune the natural wave frequency of the buoy, calculated, from eq. (10) as:

$\omega_{n}=\sqrt{S / M}$

It is known that maximum energy is captured from the incident waves when the natural frequency of the buoy is equal to the nominal incident wave frequency [11]. If the desired mass buoyancy ratio cannot be achieved through the control of $m_{b a l}$, latching $[11,12]$ (a technique which uses braking in order to artificially bring the velocity of the buoy in phase with the incident wave) can be used.

The number of RO units employed at any particular time is determined by the scheduling variable $Q_{f}$, as depicted in Fig.11. The numerical values illustrated in this are calculated like so:

$Q_{f_{\text {min }}}\left(N_{\text {ro }}\right)=\left(2.857 N_{\text {ro }} \rho_{\text {ro }}\left(\overline{P_{\text {ro }}}-P_{\text {osm }}\right)\right)$

where $Q_{f_{\min }}$ is the minimum feed water flow required to meet the $Q_{t v} / Q_{w}$ constraint. The equation is formed using this constraint, the equation $Q_{w}=\left(P_{r_{o}}-P_{o s m}\right) \rho_{r o}$ and the fact that $P_{r o}$ will be regulated at $\overline{P_{\text {ro }}}$. Hysteresis is added in the positive $Q_{f}$ direction to prevent rapid switching.

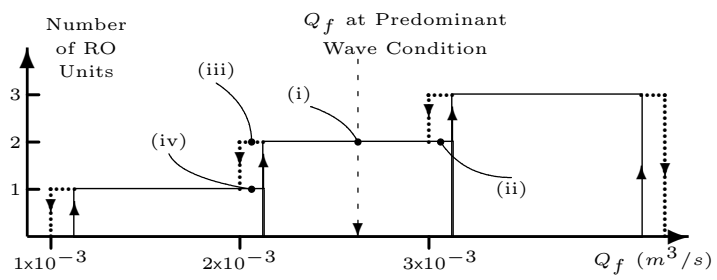

Fig. 11: RO Switching Schedule

\section{RESULTS}

A simulation model of the complete WEC system (eqs. (2), (3) and (8)), developed in MATLAB ${ }^{T M}$ using the SIMULINK package [13], is used to evaluate controller performance. Table 2 details the performance of three different pressure controllers 
at each of the various wave conditions corresponding to the points (i), (ii) (iii) and (iv) in Fig.11, with the performance measure MAD being the mean absolute deviation. For reference, a proportional controller $(\mathrm{PC})$ is hand tuned for optimum performance at the predominant wave condition. It is worth noting that, at the optimum tuning of the $\mathrm{PC}$, the valve motion consistently operates at its rate limit. The following results can be taken from Table 2 .

- SFBC with IA and DFF consistently shows the best steady state (mean) control of $P_{r o}$, and

- The SFBC with IA and DFF is the only controller to keep the variation of $P_{r o}$ within the $3 \%$ specification during all tests.

\begin{tabular}{|c||c|c|c|}
\hline \multirow{2}{*}{$\begin{array}{c}P_{r o} \\
\left(x 10^{5} \mathrm{~Pa}\right)\end{array}$} & \multicolumn{3}{c|}{ Controller } \\
\cline { 2 - 4 } & PC & SFBC + DFF & SFBC + IA + DFF \\
\hline \hline \multicolumn{3}{|c|}{ Wave condition corresponding to point (i) } \\
\hline Max & 61.327 & 61.316 & 60.962 \\
Min & 58.737 & 59.414 & 59.183 \\
Max-Min & 2.592 & 1.902 & 1.779 \\
Mean & 60.035 & 60.103 & 60.002 \\
MAD & 0.25 & 0.295 & 0.281 \\
\hline \multicolumn{3}{|c|}{ Wave condition corresponding to point (ii) } \\
\hline Max & 62.062 & 61.982 & 61.513 \\
Min & 58.646 & 59.687 & 58.430 \\
Max-Min & 3.4157 & 2.2933 & 3.083 \\
Mean & 60.09 & 60.372 & 60.001 \\
MAD & 0.353 & 0.451 & 0.521 \\
\hline \multicolumn{3}{|c|}{ Wave condition corresponding to point (iii) } \\
\hline Max & 60.680 & 60.797 & 60.628 \\
Min & 59.761 & 59.737 & 59.654 \\
Max-Min & 0.919 & 1.059 & 0.973 \\
Mean & 60.063 & 60.014 & 60.005 \\
MAD & 0.108 & 0.152 & 0.143 \\
\hline \multicolumn{3}{|c|}{ Wave condition corresponding to point (iv) } \\
\hline Max & 60.557 & 60.582 & 60.519 \\
Min & 59.794 & 59.840 & 59.725 \\
Max-Min & 0.764 & 0.741 & 0.793 \\
Mean & 60.079 & 60.017 & 60.00 \\
MAD & 0.089 & 0.083 & 0.083 \\
\hline
\end{tabular}

Table 2: Sample Set of Results for Pressure Regulation

\section{CONCLUSIONS}

The important initial step in facilitating the controller development was the establishment of a hierarchy within the control problem, with the primary focus of this paper on the pressure regulation problem. A novel decomposition of the state-space allowed a feedback controller with integral action and disturbance feed forward to be developed, with algebraic relationships between the parameters of the controller and the system parameters being maintained, allowing the controller to be scheduled across a wide variety of operating conditions.

\section{ACKNOWLEDGEMENTS}

The authors would like to thank Dr. Oliver Mason of the Hamilton Institute at NUI Maynooth and Dr. Andy McCabe of the Renewable Energy Research Group at Lancaster University, for their contribution to this work. The authors are also grateful for the financial support provided by Enterprise Ireland (under the Research Innovation Fund scheme).

\section{REFERENCES}

[1] D. Hicks, C. Please, and G. Mitcheson, "Delbuoy: Wave-powered seawater desalination system," in Proc. OCEANS, vol. 3, 1988, pp. 1049-1055.

[2] M. McCormick, P. McCabe, and D. Kraemer, "Potable water and electricity production by a hinged-barge wave energy conversion system," International Journal of Power and Energy Systems (IASTED), 1998.

[3] J. Murtagh, "Private correspondance," 2005.

[4] W. Pierson and L. Moskowitz, "A proposed spectral form for fully developed wind seas based on the similarity theory of S. A. Kitaigorodskii," Journal of Geophysical Research, vol. 69, no. 24, pp. 5181-5190, 1964.

[5] H. Eidsmoen, "On theory and simulation of heaving-buoy wave-energy converters with control," Ph.D. dissertation, University of Trondheim, Norwegian Institute of Technology, 1995.

[6] W. Cummins, "The impulse response function and ship motions," Schiffstechnik, vol. 9, pp. 101-109, 1962.

[7] "WAMIT user manual," WAMIT Inc., Chestnut Hill, Massachusetts, USA, 2002.

[8] G. Nolan, M. Ó Catháin, J. Murtagh, and J. Ringwood, "Modelling and simulation of the power take-off system for a hinge-barge wave-energy converter," in Proc. 5th European Wave Energy Conference, Cork, Ireland, September 2003, pp. 38-45.

[9] O. Mason, "Private correspondance," 2006.

[10] D. Liberzon, Switching in Systems and Control. Birkhauser, 2003.

[11] J. Falnes, Ocean Waves and Oscillating Systems. Cambridge, UK: Cambridge University Press, 2002.

[12] G. Nolan, J. Ringwood, W. Leithead, and S. Butler, "Optimal damping profiles for a heaving buoy wave energy converter," in Proc. 15th International Offshore and Polar Engineering Conference, Seoul, Korea, June 2005, pp. $477-484$.

[13] Mathworks Inc., "Using simulink (revised for simulink 6.3), simulation and model based design," Tech. Rep., 2005. 